\title{
Estimación del potencial energético del gas pobre obtenido de la gasificación del bagazo de caña de azúcar en el Perú
}

\author{
César Humberto Zavala Inga1, Victor Hugo Petrell Huamán² \\ ${ }^{1}$ Programa de Doctorado en Ciencias con Mención en Energética, Universidad Nacional de Ingeniería, \\ Facultad de Ingeniería Mecánica, Av. Tupac Amaru 210, Rímac, Lima, Perú \\ ${ }^{2}$ Laboratorio de Investigación de Procesos Termoquímicos, Universidad Nacional de Ingeniería, Facultad de \\ Petroquímica, Av. Tupac Amaru 210, Rímac, Lima, Perú
}

Recibido el 24 de noviembre del 2019. Revisado el 2 de marzo del 2020. Aceptado el 4 de marzo del 2020

DOI: https://doi.org/10.33017/RevECIPeru2020.0007/

\section{Resumen}

El propósito de este trabajo fue estimar el potencial energético del gas pobre obtenido de la gasificación del bagazo de caña de azúcar de manera computacional en gasificadores de lecho fijo de corriente descendente, a través de un modelo matemático que ha considerado el tamaño de los fragmentos de biomasa. Para este propósito, se ha tenido que determinar el área dedicada al cultivo de la caña de azúcar, la cantidad de caña de azúcar producida por cada área cultivada, la cantidad de bagazo obtenida de la caña de azúcar y la proporción de humedad y residuos secos, utilizando datos estadísticos peruanos. La humedad del bagazo debe ser inferior al $20 \%$ pero no debe estar completamente seca. Se ha considerado el modelo matemático de Prokash Roy incidiendo en las constantes del equilibrio químico y las tasas de reacciones químicas que se controlan cinéticamente a velocidad finita. Se ha utilizado una nueva codificación en el lenguaje de programación Python versión 3.x, en una plataforma interactiva llamada Jupyter Notebook. Se ha estimado que el potencial energético del gas pobre para la gasificación del bagazo de la caña de azúcar fue de 19396.6 TJ por año como valor máximo y 16843.7 TJ por año como valor mínimo.

Descriptores: Gasificación, potencial energético, gasificador de tiro descendente, residuos agrícolas.

\section{Abstract}

The purpose of this work was to estimate the energy potential of producer gas obtained from sugarcane bagasse gasification of computational way in gasifiers downdraft, through of a mathematical model that has considered the size of biomass fragments. For this purpose, we have had to determine the area dedicated to the cultivation of sugarcane, the amount of sugarcane produced by each cultivated area, the amount of bagasse obtained from sugarcane and the proportion of moisture and dry residue, using Peruvian statistical data. The humidity of the bagasse should be less than $20 \%$ but should not be completely dry. The mathematical model of Prokash Roy has been considered, having an impact on the constants of chemical equilibrium and the rates of chemical reactions that are kinetically controlled at finite speed. A new coding has been used in the Python programming language version 3.x, using an interactive platform called Jupyter Notebook. It has been estimated that the energy potential of the poor gas from the gasification of sugarcane bagasse was 19396.6 TJ per year as a maximum value and 16843.7 TJ per year as a minimum value.

Keywords: Gasification; energy potential; downdraft gasifier; agricultural residues. 


\section{Introducción}

Los desechos agrícolas, incluidos los residuos resultantes de los cultivos agrícolas [1], se componen principalmente de cáscara de plantas, hojas y tallos. La cantidad de residuos puede ser considerablemente alta, generalmente representando el doble que el producto cosechado [2]. La biomasa vegetal es una de las fuentes de energía renovables utilizadas por la humanidad desde los albores de la civilización; y actualmente se está estudiando porque las fuentes de energía fósil como el petróleo, el gas natural y el carbón son escasas y se distribuyen de manera desigual en todo el mundo. Esto crea problemas de dependencia y seguridad energética para los importadores de petróleo [3]. Las fuentes de energía fósiles también causan emisiones de gases de efecto invernadero (principalmente $\mathrm{CO}_{2}$ y $\mathrm{CH}_{4}$ ) que no se reabsorben y por ende deben mitigarse.

En las últimas dos décadas, la producción peruana de cultivos agroindustriales para exportación y uso doméstico ha crecido rápidamente. En el año 2000, la producción de cultivos de caña de azúcar fue de 7.13 millones de toneladas, creciendo a 11.13 millones de toneladas en el año 2017 [4], prácticamente duplicando la producción de caña de azúcar. Este crecimiento trae consigo la producción de grandes cantidades de desechos lignocelulósicos [5], que no siempre se utilizan de manera sostenible, causando problemas ambientales y de salud como la propagación de enfermedades en los cultivos [6]. Se obtienen varios tipos de biomasa residual de la caña de azúcar, como las hojas secas y verdes, la paja y el bagazo, que es el residuo lignocelulósico que queda después de la molienda de la caña. [4] En 2016, el bagazo de la caña de azúcar registró una producción de 2,9 millones de toneladas [7].

El uso de biomasa lignocelulósica para fines energéticos tiene grandes ventajas ambientales como la disminución de $\mathrm{CO}_{2}$, lluvia ácida, residuos, además evita la erosión y degradación de suelos. El crecimiento de la caña de azúcar absorbe $\mathrm{CO}_{2}$ del aire por fotosíntesis y fija las bajas cantidades de azufre y nitrógeno en la estructura de la planta, que reduce las emisiones de estos compuestos a la atmósfera [8]. A pesar de sus innegables ventajas, el uso de biomasa para energía, o bioenergía, también presenta algunos problemas técnicos como un alto contenido de humedad inicial, heterogeneidad en forma y tamaño, baja densidad aparente [9], esto hace que tenga menor poder calorífico comparado con otros combustibles.
Además, son fuentes altamente dispersas, lo que hace necesario llevar a cabo una evaluación exhaustiva de sus propiedades antes de definir el mejor uso como fuente de energía, ya sea directa o indirectamente [10].

Según el balance nacional de energía del Perú del 2016, el uso del bagazo para procesos de combustión directa para generadores eléctricos a representado casi el $2 \%$ [11], y se puede concluir que existe la necesidad de desarrollar aún más los procesos de biomasa energéticamente sostenible y las tecnologías de conversión. Una forma de obtener esas metas es a través de los procesos de conversión térmica de la gasificación de biomasa con aire para convertirla en un gas llamado "gas pobre o gas producto" que contiene una mezcla de gases combustibles $\left(\mathrm{CO}, \mathrm{H}_{2}\right.$ y $\left.\mathrm{CH}_{4}\right)$ y nitrógeno $\left(\mathrm{N}_{2}\right)$.

$\mathrm{Se}$ han desarrollado diferentes sistemas de gasificación de biomasa en una amplia gama de capacidades para cumplir con los requisitos de aplicaciones particulares [12-14]. La presencia de alquitrán en el gas pobre crea problemas en los motores de combustión interna para la generación de energía.

El propósito de este trabajo es simular de manera computacional el proceso dentro de un gasificador para determinar el potencial energético del gas pobre y analizar su aspecto técnico. El diseño del gasificador de lecho fijo de corriente descendente de la garganta es el más adecuado para aplicaciones de motor porque permite una mezcla máxima de gases en la región de alta temperatura, lo que ayuda a disminuir la formación de alquitrán para obtener una mayor reducción de la carbonización en el gas producto [15]. Los procesos termoquímicos que ocurren dentro de un gasificador de lecho fijo de corriente descendente son muy complejos e incluyen pirólisis, oxidación parcial y reacciones de gasificación sucesivas a temperaturas elevadas. Estos procesos dependen principalmente de la composición de la biomasa original de la planta, el contenido de humedad, la estequiometría de la reacción y el diseño del gasificador.

Por lo tanto, un modelo adecuado para el gasificador de lecho fijo de corriente descendente puede predecir los parámetros de rendimiento óptimos para su funcionamiento. Sin embargo, varios investigadores han informado sobre el comportamiento termoquímico [16-21] basado en un modelo de equilibrio termodinámico, pero este tipo de modelos no representa un proceso real de gasificación, pero si un equilibrio químico en la zona 
de pirooxidación y reacciones químicas cinéticamente controladas a velocidad finita en la zona de reducción y esto implica ayuda computacional con una serie de algoritmos complejos [22-24].

Python es un lenguaje de alto nivel ya que contiene en forma implícitas algunas estructuras de datos como listas, diccionarios, conjuntos y tuplas, que permiten realizar algunas tareas complejas en pocas líneas de código y de manera legible [25]. Jupyter notebook es una aplicación cliente-servidor. La aplicación inicia el servidor en la máquina local y abre la interfaz del portátil en el navegador web desde donde se puede editar y ejecutar [26].

Los modelos cinéticos se basan en la ruta de reacción química prescrita por Wang y Kinoshita [27] para la reducción de char de la biomasa en la zona de reducción del gasificador. Para estimar el potencial energético del gas pobre obtenido de la gasificación del bagazo de caña de azúcar por año [28]; se propuso comenzar a trabajar con el modelo de gasificación de Prokash Roy [29], por utilizar una matemática no tan compleja con un punto de vista fisicoquímico aceptable para estimar la energía.

Este modelo fue validado con los datos experimentales de Jayah [30]. Al utilizar el modelo de Roy [29] con fragmentos de biomasa de tamaño $4.4 \mathrm{~cm}$ y $5.5 \mathrm{~cm}$ las predicciones de las componentes del gas pobre se alejaban a los valores reales de la data experimental [30].

Por lo tanto, se tuvo la necesidad de realizar cambios en las constantes de equilibrio químico y a la velocidad cinética de las reacciones de metanización y cambio de agua-gas (wáter-gas shift). De esta manera se ha obtenido valores de concentración de gases más cercanas a la experimental; y cuyos porcentajes de error de la validación, se encuentran por debajo de los calculados con el modelo de Prokash Roy. Para obtener los resultados numéricos se usó el lenguaje de programación Python con una nueva codificación.

\section{Caracterización de la biomasa.}

La biomasa utilizada en el estudio experimental de gasificación es el bagazo de caña de azúcar, este combustible de biomasa es un residuo agrícola obtenido de la Empresa Agroindustrial Pomalca S.A.A. ubicado en el distrito de Pomalca, provincia de Chiclayo, Perú. Las muestras de bagazo de caña de azúcar (SCB) se secaron en un espacio ventilado pero cubierto durante 15 días hasta que la humedad fue inferior al $10 \%$, luego se molieron en un molino de cuchillas con un tamaño de partícula inferior a $850 \mu \mathrm{m}$. La muestra se almacenó en un recipiente hermético y en un ambiente ventilado para su uso posterior. La determinación experimental de los datos de análisis elemental del SCB requiere una instrumentación especial, mientras que los datos de análisis próximo se pueden obtener fácilmente mediante el uso de equipos comunes.

\subsection{Análisis elemental}

El análisis de carbono, hidrógeno y nitrógeno $(\mathrm{C}, \mathrm{H}$, N) se realizó de acuerdo con los estándares ASTM D5373-Método A y ASTM D4239, utilizando un analizador elemental LECO, serie modelo 628, para el análisis de carbono, hidrógeno y nitrógeno. $(\mathrm{C}, \mathrm{H}$, N) El análisis de azufre se llevó a cabo en el Módulo adicional de azufre de la serie 628 y el contenido de oxígeno se obtuvo por diferencia. Trabajamos con muestras previamente secadas a $105^{\circ} \mathrm{C}$ durante $24 \mathrm{~h}$ y con un tamaño de partícula menor de $250 \mu \mathrm{m}$. Todo el trabajo analítico se realizó por triplicado y los resultados se informan como muestras secas.

\subsection{Análisis próximo}

El análisis próximo se realizó por termogravimetría de acuerdo con el estándar ASTM D7582, utilizando un analizador termogravimétrico Perkin Elmer TGA 4000; la rampa de calentamiento utilizada fue de temperatura ambiente a $105^{\circ} \mathrm{C}$ a una velocidad de calentamiento de $15^{\circ} \mathrm{C} / \mathrm{min}$, seguida de una isoterma a $105^{\circ} \mathrm{C}$ durante 80 minutos, luego una rampa de calentamiento de $105^{\circ} \mathrm{C}$ a $950^{\circ} \mathrm{C}$ a velocidad de calentamiento de $30^{\circ} \mathrm{C} / \mathrm{min}$, seguido de una isoterma a $950^{\circ} \mathrm{C}$ durante 7 minutos. Todas estas rampas se hicieron bajo un flujo de nitrógeno de $70 \mathrm{~cm}^{3} / \mathrm{min}$. Para la determinación de cenizas, la rampa de calentamiento fue de temperatura ambiente a $500^{\circ} \mathrm{C}$ a una velocidad de calentamiento de $8^{\circ} \mathrm{C} / \mathrm{min}$ bajo un flujo de oxígeno de $70 \mathrm{~cm}^{3} / \mathrm{min}$.

En todos los casos trabajamos con un tamaño de partícula menor de $250 \mu \mathrm{m}$. Todas las muestras se mantuvieron en un desecador durante seis horas antes del análisis. Se ejecutó un mínimo de dos réplicas por cada prueba.

\subsection{Valor calorífico superior}

El valor calorífico superior (HHV) se determinó de acuerdo con el ASTM D5865; se tomaron de 0.5 a 1 $\mathrm{g}$ de biomasa pulverizada de un tamaño inferior a $850 \mu \mathrm{m}$ y se quemaron en una bomba calorimétrica 
adiabática (PARR1341) en condiciones controladas. El HHV se calculó a partir de las observaciones de temperatura realizadas antes y después de la combustión. El HHV obtenido se informa como base recibida; se realizaron tres de cada prueba para garantizar la repetibilidad de la prueba. Los resultados del análisis próximo, elemental y HHV del bagazo de caña de azúcar (SCB); se muestran en las tablas 1 y 2 , donde también se muestran los valores obtenidos en otras investigaciones. Los elementos carbono, hidrógeno, nitrógeno y azufre están dentro del rango dado por las referencias, pero en el caso del oxígeno se obtiene un valor más bajo.

Este último es un resultado positivo, ya que valores más bajos de oxígeno significan un valor calorífico más alto, un mejor uso como combustible o una mejor carga para procesos de conversión termoquímica adicionales. La biomasa analizada tiene pequeños valores de elementos de nitrógeno y azufre que son precursores de NOx y SOx cuando ocurre el proceso de combustión.

\begin{tabular}{|c|c|}
\hline \multicolumn{2}{|c|}{ Nomenclatura } \\
\hline$C$ & Carbón (\% masa). \\
\hline$H$ & Hidrógeno (\% masa). \\
\hline$O$ & Oxígeno (\% masa). \\
\hline$N$ & Nitrógeno (\% masa). \\
\hline$F C$ & Carbon fijo (\% masa). \\
\hline ASH & $\begin{array}{l}\text { Ceniza del análisis elemental del combustible seco } \\
(\% \text { masa })\end{array}$ \\
\hline MC & Contenido de humedad (\% masa). \\
\hline $\boldsymbol{F}$ & Velocidad de flujo de combustible seco (Kg/h). \\
\hline $\boldsymbol{A}$ & Velocidad de flujo de aire seco (Kg/h). \\
\hline$\Delta V_{k}$ & volume control k. \\
\hline$i$ & Especies. \\
\hline out & Salida. \\
\hline in & Ingreso. \\
\hline$T_{o}$ & Temperature a $298 \mathrm{~K}$. \\
\hline $\boldsymbol{x}_{i}$ & Numero de moles de $\mathrm{i}$. \\
\hline $\boldsymbol{\theta}$ & Angulo de divergencia de la zona de reducción. \\
\hline$a$ & Numero de moles de oxígeno por mol de biomasa. \\
\hline$w$ & Numero de moles de agua por mol de biomasa. \\
\hline $\boldsymbol{X}$ & Velocidad de flujo molar (mol/s). \\
\hline$y_{i}$ & Fracción molar de i. \\
\hline$t$ & Toneladas. \\
\hline$R_{i}^{k}$ & $\begin{array}{l}\text { Velocidad neta de formación de las especies i en el volumen control } \\
\mathrm{k}\left(\mathrm{mol} / \mathrm{m}^{3} . \mathrm{s}\right) \text {. }\end{array}$ \\
\hline$\Delta V_{k}$ & Diferencial del volumen control $\mathrm{k}, \mathrm{m}^{3}$ \\
\hline$T$ & Temperature $(\mathrm{K})$. \\
\hline$T_{a}$ & Temperatura del aire al ingresar al gasificador. \\
\hline$H^{\prime}$ & Altura de la zona de reducción (m). \\
\hline$E_{R i}$ & Energía de activación $\mathrm{R}_{\mathrm{i}},(\mathrm{J} / \mathrm{mol})$. \\
\hline$A_{R i}$ & Factor Pre-exponencial de la reacción $\mathrm{R}_{\mathrm{i}}\left(\mathrm{mol} / \mathrm{m}^{3}\right.$. s). \\
\hline CRF & Factor de reactividad del CHAR. \\
\hline$\overline{\bar{M}}_{i}$ & Masa molecular de i. \\
\hline $\boldsymbol{R}$ & Constante universal de gases (J/mol.K). \\
\hline
\end{tabular}

Los valores reportados de la composición elemental son consistentes con los reportados en estudios previos. El contenido de materia volátil (VM) indica que los componentes se eliminan de la biomasa cuando se calienta a altas temperaturas. Estos compuestos gaseosos pueden ser combustibles (gases $\left.\mathrm{C}_{\mathrm{x}} \mathrm{H}_{\mathrm{y}}, \mathrm{CO} \circ \mathrm{H}_{2}\right)$ y no combustibles $\left(\mathrm{CO}_{2}, \mathrm{SO}_{2}\right.$, $\mathrm{NO}_{\mathrm{x}}$ ) [8]. Los valores altos de este parámetro facilitan la pirólisis de biomasa.

La ceniza $(\mathrm{ASH})$ es el componente inorgánico de la biomasa que permanece después de la combustión [8], por lo que su composición y sus variedades dependen según el tipo de biomasa, las condiciones del suelo y el método de cultivo [32]. Los valores de ceniza encontrados son relativamente altos para el bagazo de caña de azúcar.

Las cenizas pueden causar problemas de ensuciamiento y formación de escoria [31]. El contenido de carbono fijo (FC) es la masa que queda después de que la materia volátil ha sido expulsada de la biomasa, sin contar el contenido de cenizas y humedad (MC), esta es la zona más resistente a la degradación térmica [8].

\section{Descripción del modelo}

De acuerdo con la ecuación. (1), el modelo matemático para la estimación del potencial energético del gas pobre de bagazo de caña de azúcar en Perú ( $\left(E P_{S C B}, T J / a \tilde{n} o\right)$ consta de 2 partes. La primera parte es calcular la masa de residuos secos de bagazo de caña de azúcar para el año $2017\left(M_{d r}\right.$, t/año) y la segunda es el máximo valor calorífico inferior más óptimo por tonelada $(E, T J / t)$ que se obtiene utilizando el modelo de la gasificación de biomasa con contenido de humedad (según la base recibida).

Donde:

$$
E P_{S C B}=M_{d r} . E
$$$$
M_{d r}=\left(S \cdot R_{c} \cdot F_{r} \cdot Y_{r s}\right)
$$

En la ecuación (2), establece la relación entre las variables más relevantes para estimar la masa de residuos secos $\left(M_{d r}\right)$, como el número de hectáreas correspondientes al área cultivada (S), el rendimiento del producto principal en toneladas por hectárea plantada $\left(R_{c}\right)$, la masa de residuos de bagazo en toneladas $\left(F_{r}\right)$ obtenida del producto principal, y la relación entre la masa de residuos húmedos y secos $\left(Y_{r s}\right)$, teniendo en cuenta que para estimar el potencial energético de la biomasa agrícola es importante que el bagazo de caña de azúcar contenga una cantidad de humedad lo más bajo posible. 
Tabla 1: Análisis elemental, análisis próximo, valor calorífico de SCB en Perú.

\begin{tabular}{|c|c|c|}
\hline Tipo de biomasa & \multicolumn{2}{|c|}{$\begin{array}{c}\text { Bagazo de caña de } \\
\text { azúcar (SCB) }\end{array}$} \\
\hline \multirow{5}{*}{$\begin{array}{c}\text { Análisis elemental } \\
\text { (base seca) } \\
(\%)\end{array}$} & $C$ & 45.13 \\
\hline & $H$ & 6.37 \\
\hline & $N$ & 0.21 \\
\hline & $S$ & 0.05 \\
\hline & $O^{a}$ & 41.07 \\
\hline \multirow{4}{*}{$\begin{array}{c}\text { Análisis próximo } \\
(\%)\end{array}$} & $M C$ & 1.8 \\
\hline & ASH & 7.04 \\
\hline & $V M$ & 77.7 \\
\hline & $F C^{b}$ & 13.38 \\
\hline \multirow{2}{*}{$\begin{array}{c}\text { Valor calorífico } \\
\text { (MJ/Kg) }\end{array}$} & HHV & 18.28 \\
\hline & $L H V$ & 16.95 \\
\hline
\end{tabular}

${ }^{a}$ Oxigeno\% $=100-\% \mathrm{C}-\% \mathrm{H}-\% \mathrm{~N}-\% \mathrm{~S}-\%$ Ash (base seca)

${ }^{\mathrm{b}} \mathrm{FC}$; \% carbón fijo se obtuvo por diferencia.

Tabla 2: Valores referenciales de análisis elemental de biomasa SCB, análisis aproximado y valor calorífico.

\begin{tabular}{|c|c|c|}
\hline Tipo de biomasa & Bag & $\begin{array}{l}\text { Ĩ̃a de azúcar } \\
\text { CB) }\end{array}$ \\
\hline \multirow{5}{*}{$\begin{array}{c}\text { Análisis elemental } \\
\text { (base seca) } \\
(\%)\end{array}$} & $C$ & $\begin{array}{l}44.8^{a} \\
45.3^{b}\end{array}$ \\
\hline & $H$ & $\begin{array}{r}5.87 a \\
6.8 b\end{array}$ \\
\hline & $N$ & $\begin{array}{l}0.24^{a} \\
0.5^{b}\end{array}$ \\
\hline & $S$ & $\begin{array}{c}0.06^{a} \\
0.3^{b}\end{array}$ \\
\hline & $O^{a}$ & $\begin{array}{r}48.97^{a} \\
47.1^{b} \\
\end{array}$ \\
\hline \multirow{4}{*}{$\begin{array}{c}\text { Análisis próximo } \\
(\%)\end{array}$} & MC & $\begin{array}{l}5.4^{a} \\
n r^{b}\end{array}$ \\
\hline & $A S H$ & $\begin{array}{r}3.1^{a} \\
n r^{b}\end{array}$ \\
\hline & $V M$ & $\begin{array}{l}80.2^{a} \\
2.31^{b}\end{array}$ \\
\hline & $F C^{b}$ & $\begin{array}{l}11.3^{a} \\
7.16^{b}\end{array}$ \\
\hline $\begin{array}{c}\text { Valor calorífico } \\
\text { (MJ/Kg) }\end{array}$ & $H H V$ & $\begin{array}{c}18.0^{a} \\
17.88^{b}\end{array}$ \\
\hline
\end{tabular}

$\mathrm{nr}=$ No se informa en la referencia

\subsection{Cálculo de la cantidad total de biomasa seca}

La información estadística peruana extraída de la referencia [4] se recopila y se muestra en la tabla 3. Además, los factores de crecimiento para la producción y el área sembrada se calcularon para los años 2000 y 2017.

La relación entre la cantidad de residuos con contenido de humedad y el área sembrada, tiene un valor promedio (2012-2016) de $20.32 \mathrm{t} /$ ha para el año 2017. De esta manera, fue posible calcular la cantidad de biomasa con contenido de humedad. para desarrollar las variables que se muestran en la tabla 4.
Tabla 3: Factor de crecimiento de la producción de caña de azúcar y el área sembrada en el Perú.

\begin{tabular}{|c|c|c|c|}
\hline $\begin{array}{c}\mathbf{R}_{\mathrm{c}} \\
\text { (t de PP/Ha) }\end{array}$ & $\begin{array}{c}\mathbf{F}_{\mathbf{r}} \\
\text { ( t de BH /t de PP) }\end{array}$ & $\begin{array}{c}\mathbf{Y}_{\text {rs }} \\
\text { (t de BS /t de } \\
\text { BH })\end{array}$ & $\begin{array}{c}\mathbf{M}_{\text {dr }} \\
\text { ( t de BS /año) }\end{array}$ \\
\hline 105.7 & 0.28 & $0.588^{\mathrm{a}}$ & 1815280.0 \\
\hline
\end{tabular}

BS: Biomasa seca, BH: biomasa húmeda y $\mathrm{PP}$ : producto principal. a [25]

Tabla 4: Cantidad de masa residual seca de SCB en Perú, en el año 2017.

\begin{tabular}{|c|c|c|c|c|c|c|}
\hline Planta & \multicolumn{2}{|c|}{$\begin{array}{c}\text { Producto principal } \\
\text { (t/año) }\end{array}$} & \multirow{2}{*}{$\begin{array}{c}\text { Factor de } \\
\text { crecimiento } \\
\text { de }\end{array}$} & \multicolumn{2}{|c|}{$\begin{array}{c}\text { Area sembrada } \\
\text { (Ha/año) }\end{array}$} & $\begin{array}{c}\text { Factor de } \\
\text { crecimiento } \\
\text { de la zona } \\
\text { producción }\end{array}$ \\
& 2000 & 2017 & 2000 & 2017 & \\
\hline $\begin{array}{c}\text { Caña } \\
\text { se } \\
\text { azúcar }\end{array}$ & 7135154 & 11127473 & 1.6 & 63808 & 105296 & 1.7 \\
\hline
\end{tabular}

La información estadística peruana extraída de la referencia [4] se recopila y se muestra en la tabla 3. Además, los factores de crecimiento para la producción y el área sembrada se calcularon para los años 2000 y 2017. La relación entre la cantidad de residuos con contenido de humedad y el área sembrada, tiene un valor promedio (2012-2016) de $20.32 \mathrm{t} /$ ha para el año 2017. De esta manera, fue posible calcular la cantidad de biomasa con contenido de humedad. para desarrollar las variables que se muestran en la tabla 4.

\subsection{Cálculo de energía por toneladas de biomasa seca}

El diagrama esquemático de un gasificador de tiro descendente y la ampliación de la zona de reducción se muestran en la Figura 1. Las dimensiones del gasificador de tiro descendente en este estudio se muestra en la Tabla 5 , que son iguales a las de la literatura de Jayah et al. [30].

El modelo se basa en el modelo de gasificación de Roy [29], con algunas modificaciones en la constante de equilibrio de la reacción de metanización y en la velocidad cinética con respecto a las reacciones de Boudouard y el cambio de aguagas (Water -Shift) cuando el tamaño del fragmento de la biomasa vegetal está cambiando. De esta manera, se reduce el error promedio de la composición de gases secos en el momento de la validación.

El modelado en la zona de oxidación se resuelve con base en consideraciones de equilibrio químico, ya que las reacciones ocurren a una temperatura razonablemente alta. La zona de reducción está modelada en base a la cinética química del carbón, considerando una gran cantidad de volúmenes de control elemental a lo largo de esta zona. 
El balance de masa de cada especie y los balances de energía se han realizado en cada volumen de control considerando la tasa finita de reacciones químicas. Las contribuciones de las cenizas en el combustible y en la pérdida de calor del gasificador de tiro descendente se han considerado en la ecuación del balance de energía.

Se prefiere la biomasa vegetal con baja humedad debido a su mayor valor calorífico bruto y porque demasiada humedad podría resultar en una gasificación ineficaz debido a una temperatura baja.

Además, hará que sea difícil que las reacciones de reducción tengan lugar de manera efectiva y pueda no ser viable desde el punto de vista tecnológico.

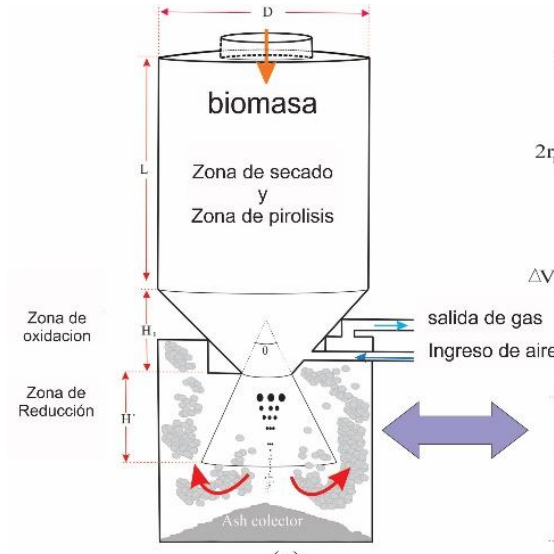

(a)

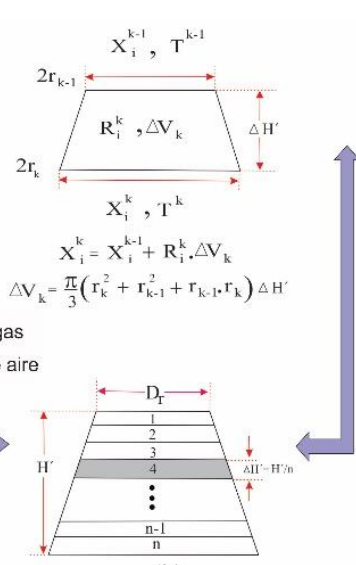

(b)
Figura 1: Diagrama esquemático de (a) gasificador de lecho fijo de corriente descendente y (b) ampliación de la zona de reducción.

Tabla 5: Dimensiones del gasificador de lecho fijo de corriente descendente.

\begin{tabular}{|c|c|}
\hline $\begin{array}{c}\text { Dimensiones } \\
\left(\text { para } \theta=120^{\circ}\right)\end{array}$ & $\begin{array}{l}\text { Medida } \\
(\mathrm{mm})\end{array}$ \\
\hline $\mathrm{D}$ & 920 \\
\hline $\mathrm{L}$ & 1150 \\
\hline $\mathrm{H}_{1}$ & 740 \\
\hline $\mathrm{H}^{\prime}$ & 220 \\
\hline $\mathrm{D}_{\mathrm{r}}$ & 100 \\
\hline
\end{tabular}

La reacción global en la zona de oxidación de la pirólisis se puede escribir como:

\footnotetext{
$\mathrm{CH}_{m} \mathrm{O}_{n}+w \mathrm{H}_{2} \mathrm{O}+\mathrm{aO}_{2}+3.76 \mathrm{aN}_{2} \rightarrow x_{1} \mathrm{H}_{2}+x_{2} \mathrm{CO}+x_{3} \mathrm{CO}_{2}+$

$x_{4} \mathrm{H}_{2} \mathrm{O}+x_{5} \mathrm{CH}_{4}+x_{6} \mathrm{~N}_{2}+x_{7} \mathrm{C}$
}

El contenido de humedad de la biomasa vegetal se define como

$$
M C=\left(\frac{\text { Mass }_{\text {water }}}{\text { Mass }_{\text {water }}+\text { Mass dried bagasse }}\right)
$$

Por lo tanto, la cantidad de agua en términos de contenido de humedad de la biomasa puede determinarse por:

$$
w=\left(\frac{0.01 * M C}{(1-0.01 * M C)(1-0.01 * A S H)}\right)\left(\frac{\bar{M}_{F U E L}}{\bar{M}_{H_{2} O}}\right)
$$

Tabla 6: Composición de residuos de madera de caucho después de la referencia.

\begin{tabular}{|c|c|c|c|}
\hline Variable & $\begin{array}{c}\text { Como base } \\
\text { recibida } \\
\text { (ARB) } \\
\text { (\% masa) }\end{array}$ & $\begin{array}{c}\text { Base seca } \\
\text { (\%masa) }\end{array}$ & $\begin{array}{c}\text { Ceniza libre de } \\
\text { humedad } \\
\text { (DAF) } \\
\text { (\%masa) }\end{array}$ \\
\hline $\boldsymbol{C}$ & 42.504 & 50.6 & 50.942 \\
\hline $\boldsymbol{H}$ & 5.46 & 6.5 & 6.544 \\
\hline $\boldsymbol{O}$ & 35.2884 & 42.2 & 42.514 \\
\hline $\boldsymbol{A S H}$ & 0.1848 & 0.7 & n.c. \\
\hline $\boldsymbol{M C}$ & 16 & n.c. & n.c. \\
\hline Total & 100 & 100 & 100 \\
\hline n.c: no se considera & \multicolumn{2}{l}{} \\
\end{tabular}

La relación de equivalencia $(E R)$ se define como la relación de la fracción estequiométrica a la fracción del aire-combustible actual, $\left(\frac{A}{F}\right)_{\text {actual }}$ es un dato tomado de la referencia [30].

ER $=\frac{\left(\frac{A}{F}\right)_{\text {estequiometrico }}}{\left(\frac{A}{F}\right)_{\text {actual }}}=\frac{\frac{28.84}{21}\left(\left(\left(C_{D A F} * \frac{0.01}{12.011}\right)+\left(H_{D A F} * \frac{0.01}{4} * 1.008\right)-\left(O_{D A F} * \frac{0.01}{32}\right)\right)\right)}{\left(\frac{A}{F}\right)_{\text {actual }}}$

Donde DAF es la cantidad de cenizas libre de humedad. Los balances de masas para $\mathrm{C}, \mathrm{H}, \mathrm{O}$ y $\mathrm{N}$ se calculan de la siguiente manera:

$$
\begin{gathered}
x_{2}+x_{3}+x_{5}+x_{7}=1 \\
m+2 w=2 x_{1}+2 x_{4}+4 x_{5} \\
n-w+2 a=x_{2}+2 x_{3}+4 x_{4} \\
3.76 a=x_{6} \\
C=\frac{F C}{\left(x_{5}+x_{7}\right)}
\end{gathered}
$$

Donde FC es el carbono fijo en la biomasa en base seca. Las dos reacciones de equilibrio en la zona de pirólisis-oxidación son:

\section{Reacción de cambio de agua-gas:}

$\mathrm{CO}+\mathrm{H}_{2} \mathrm{O} \rightleftharpoons \mathrm{CO}_{2}+\mathrm{H}_{2} \quad-41 \mathrm{~kJ} / \mathrm{mol}$

\section{Reacción de metanización:}

$\mathrm{C}+2 \mathrm{H}_{2} \rightleftharpoons \mathrm{CH}_{4}$

$-74.8 \mathrm{~kJ} / \mathrm{mol}$

Las constantes de equilibrio para ellos son:

$$
\begin{aligned}
& K_{1}=\frac{x_{3} \cdot x_{1}}{x_{2} \cdot x_{4}}=e^{\left(-g_{T, \mathrm{CO}_{2}}^{0}-g_{T, \mathrm{H}_{2}}^{0}+g_{T, C O}^{0}+g_{T, \mathrm{H}_{2} \mathrm{O}}^{0}\right) /} / R T \\
& K_{2}=\frac{x_{5}}{x_{1}^{2}} \cdot n_{t}=e^{\left(-g_{T, C H_{4}}^{0}+2 g_{T, H_{2}}^{0}\right) /} / R T
\end{aligned}
$$


Tabla 7: Entalpias y coeficientes empíricos.

\begin{tabular}{|c|c|l|l|l|l|l|l|l|}
\hline $\begin{array}{c}\text { Compuesto } \\
(\boldsymbol{j})\end{array}$ & $\begin{array}{c}\boldsymbol{h}_{\boldsymbol{f} \boldsymbol{j}}^{\boldsymbol{j}} \\
\boldsymbol{k J} / \mathbf{m o l}\end{array}$ & \multicolumn{1}{|c|}{$\boldsymbol{a}^{\prime}$} & $\boldsymbol{b}^{\prime}$ & \multicolumn{1}{c|}{$\boldsymbol{c}^{\prime}$} & $\boldsymbol{d}^{\prime}$ & $\boldsymbol{e}^{\prime}$ & $\boldsymbol{f}^{\prime}$ & $\boldsymbol{g}^{\prime}$ \\
\hline $\mathbf{C O}$ & -110.5 & $5.619 \times 10^{-3}$ & $-1.190 \times 10^{-5}$ & $6.383 \times 10^{-9}$ & $-1.846 \times 10^{-12}$ & $-4.891 \times 10^{2}$ & $8.684 \times 10^{-1}$ & $-6.131 \times 10^{-2}$ \\
\hline $\mathbf{C O}_{\mathbf{2}}$ & -393.5 & $-1.949 \times 10^{-2}$ & $3.122 \times 10^{-5}$ & $-2.448 \times 10^{-8}$ & $6.946 \times 10^{-12}$ & $-4.891 \times 10^{2}$ & 5.270 & $-1.207 \times 10^{-1}$ \\
\hline $\mathbf{H}_{\mathbf{2}} \mathbf{O}$ & -241.8 & $-8.950 \times 10^{-3}$ & $-3.672 \times 10^{-6}$ & $5.209 \times 10^{-9}$ & $-1.478 \times 10^{-12}$ & 0.0 & 2.868 & $-1.722 \times 10^{-2}$ \\
\hline $\mathbf{C H}_{\mathbf{4}}$ & -74.8 & $-4.620 \times 10^{-2}$ & $1.130 \times 10^{-5}$ & $1.319 \times 10^{-8}$ & $-6.647 \times 10^{-12}$ & $-4.891 \times 10^{2}$ & $1.411 \times 10$ & $-2.234 \times 10^{-1}$ \\
\hline
\end{tabular}

Donde $n_{t}=\sum_{i=1}^{6} x_{i}$, son los moles totales de gas húmedo, y $g_{f, T, j}^{o}$ representa la función estándar de formación de Gibbs a la temperatura $T$ dada de la especie de gas $j$ y $h_{f, j}^{o}$ es la entalpía de la formación, que se puede expresar mediante la ecuación empírica a continuación:

$$
g_{f, T, j}^{o}=h_{f, j}^{o}-a^{\prime} T L n(T)-b^{\prime} T^{2}-\left(\frac{c^{\prime}}{2}\right) T^{3}-\left(\frac{d^{\prime}}{3}\right) T^{4}+\left(\frac{e^{\prime}}{2 T}\right)+f^{\prime}+g^{\prime} T
$$

Los valores de los coeficientes $a^{\prime}-g^{\prime}$ y la entalpía de formación de los gases se presentan en la Tabla 7 [33].

La variación del calor específico de las especies gaseosas con la temperatura se ha contabilizado como:

$C_{p}^{o}(T)=R *\left(a_{1}+a_{2} \cdot T+a_{3} \cdot T^{2}+a_{4} \cdot T^{3}+a_{4} \cdot T^{4}\right)$, en $J / m o l . K$,

Los valores de $a_{1}, a_{2}, a_{3}, a_{4}$ y $a_{5}$ se han tomado de las tablas termodinámicas de la NASA [34].

Sin embargo, se han considerado valores constantes de calor específico para carbón (23.4 J / mol.K) [35] y cenizas (0.84 J / g.K) [36].

En el balance energético, la pérdida de calor se expresa como:

$Q_{\text {loss }}=m_{A S H} \cdot C_{p_{A S H}}\left(T-T_{O}\right)+\sum_{i=1}^{6} x_{i}\left(h_{f_{i}}^{o}+\int_{T_{o}}^{T} C_{p_{i}} d T\right)+x_{7} C_{p_{C H A R}}\left(T-T_{o}\right)-$

$\left(h_{f_{\text {wood }}}^{o}+a \int_{T_{o}}^{T_{a}} C_{p_{O_{2}}} d T+3.76 a \int_{T_{o}}^{T_{a}} C_{p_{N_{2}}} d T+w h_{f_{H_{2} O}}\right)$

A medida que se pierde el calor de la zona de pirooxidación del gasificador, el valor de Qloss es termodinámicamente negativo. La solución simultánea de la ecuación. ( 7 a 11) y las ecuaciones (14) y (15), junto con la ecuación (18), se han realizado para evaluar la composición y la temperatura del producto a la salida de la zona de oxidación. La participación de las especies gaseosas ( $i=1$ a 6 ), el carbón y las cenizas en la corriente de energía de salida se tienen en cuenta por separado. La zona de reducción para el cálculo se muestra en la Fig. 1 (b).

Las cuatro reacciones de reducción consideradas en la zona de reducción son:
R1 (reacción de Boudouard):

$$
C_{(s)}+\mathrm{CO}_{2(g)} \rightleftharpoons 2 \mathrm{CO}_{(g)} \quad+172 \mathrm{~kJ} / \mathrm{mol}
$$

R2 (reacción de cambio de agua-gas):

$$
\mathrm{C}_{(\mathrm{s})}+\mathrm{H}_{2} \mathrm{O}_{(\mathrm{g})} \rightleftharpoons \mathrm{CO}_{(\mathrm{g})}+\mathrm{H}_{2(\mathrm{~g})}+131 \mathrm{~kJ} / \mathrm{mol}
$$

R3 (reacción de metanización):

$$
\mathrm{C}_{(\mathrm{s})}+2 \mathrm{H}_{2(\mathrm{~g})} \rightleftharpoons \mathrm{CH}_{4(\mathrm{~g})} \quad-74.8 \mathrm{~kJ} / \mathrm{mol}
$$

R4 (reacción de reforma de vapor):

$$
\mathrm{CH}_{4(g)}+\mathrm{H}_{2} \mathrm{O}_{(\mathrm{g})} \rightleftharpoons \mathrm{CO}_{(\mathrm{g})}+3 \mathrm{H}_{2(\mathrm{~g})} \quad+206 \mathrm{~kJ} / \mathrm{mol}
$$

Las tasas de reacción volumétrica de estas reacciones químicas se han expresado como se informa en [29,37]. Adoptamos un factor de reactividad de carbón constante (CRF) de 100, las constantes cinéticas de los parámetros se pueden encontrar en la Tabla 8, tomada de [38].

Tabla 8: Constantes cinéticas de las reacciones de reducción.

\begin{tabular}{|c|c|c|}
\hline Reacción R & $\begin{array}{c}\boldsymbol{E}_{\boldsymbol{R}} \\
(\boldsymbol{J} / \mathbf{m o l})\end{array}$ & $\boldsymbol{A}_{\boldsymbol{R}}$ \\
\hline $\mathbf{1}$ & 77390 & 36.16 \\
\hline $\mathbf{2}$ & 121620 & $1.517 \times 10^{4}$ \\
\hline $\mathbf{3}$ & 19210 & $4.189 \times 10^{-3}$ \\
\hline $\mathbf{4}$ & 36150 & $7.301 \times 10^{-2}$ \\
\hline
\end{tabular}

$$
\begin{aligned}
& r_{R 1}=A_{1} * C R F * e^{\left(\frac{-E_{R 1}}{R T}\right)} *\left(y_{C O_{2}}-\frac{y_{C O}^{2}}{K_{R 1}}\right) \\
& r_{R 2}=A_{2} * C R F * e^{\left(\frac{-E_{R 2}}{R T}\right)} *\left(y_{H_{2} O}-\frac{y_{H_{2}} y_{C O}}{K_{R 2}}\right) \\
& r_{R 3}=A_{3} * C R F * e^{\left(\frac{-E_{R 3}}{R T}\right)} *\left(y_{H_{2}}^{2}-\frac{y_{C H_{4}}}{K_{R 3}}\right) \\
& r_{R 4}=A_{4} * C R F * e^{\left(\frac{-E_{R 4}}{R T}\right)} *\left(y_{H_{2} O} y_{C H_{4}}-\frac{y_{H_{2}} y_{C O}}{K_{R 4}}\right)
\end{aligned}
$$

Donde $y_{i}$ es fracción molar. Las constantes de equilibrio $K_{R}$ para las reacciones $R 1$ a $R 4$ se calculan de la siguiente manera:

$$
\begin{aligned}
& K_{R 1}=e^{\frac{\left(-2 g_{f, T, C O}^{o}+g_{f, T, C O_{2}}^{o}\right)}{R T}} \\
& K_{R 2}=e^{\frac{\left(-g_{f, T, C O}^{o}-g_{f, T, H_{2}}^{o}+g_{f, T, H_{2} O}^{o}\right)}{R T}} \\
& K_{R 3}=e^{\frac{\left(-g_{\left.f, T, C H_{4}+2 g_{f, T, H}\right)}^{o}\right.}{R T}}
\end{aligned}
$$


$K_{R 4}=e^{\frac{\left(-g_{f, T, C O}^{o}-3 g_{f, T, H_{2}}^{o}+g_{f, T, C H_{4}}^{o}+g_{f, T, H_{2} O}^{o}\right)}{R T}}$

Dado que la zona de reducción del gasificador de lecho fijo de corriente descendente se ha dividido en volúmenes de control a lo largo de su altura, el balance de masa para la especie / en el volumen control $k$ se puede expresar como:

$$
X_{l}^{k}=X_{l}^{k-1}+\left(R_{l}^{k}\right)\left(\Delta V_{k}\right) ; \text { para } I=1 \text { al } 7
$$

Donde $R_{l}^{k}$ puede ser, por ejemplo $R_{H_{2} O=}^{k}-r_{R 2}-r_{R 4}$, $R_{C O_{2}}^{k}=-r_{R 1}$, etc.

La temperatura en un volumen control de la zona de reducción se calcula utilizando el balance de energía, donde se desprecia la pérdida de calor $Q_{\text {loss }}^{K}$.

$\sum_{i=1}^{6} X_{i}^{K-1}\left(h_{f, i}^{o}+\int_{T_{o}}^{T^{k-1}} C_{p, i} d T\right)+X_{7}^{K-1} \cdot C p_{C}\left(T^{k-1}-T_{o}\right)+m_{A S H} C_{p, A S H}$

$=\sum_{i=1}^{6} X_{i}^{K}\left(h_{f, i}^{o}+\int_{T_{o}}^{T^{k}} C_{p_{i}} d T\right)+X_{7}^{K} \cdot C p_{C}\left(T^{k}-T_{o}\right)+m_{A S H} C_{p, A S H}\left(T^{k}-T_{o}\right)$

El valor calorífico inferior (LHV) del gas pobre se determina a partir de la composición química del gas y LHV de componentes individuales. [38]

$L H V_{D G}=\left(0.01 * 12.622 * \mathrm{Y}_{C O}+0.01 * 10.788 * \mathrm{Y}_{H_{2}}+0.01 * 35.814 * Y_{C H_{4}}\right) ;$ en $\mathrm{MJ} / \mathrm{m}^{3}$

Donde Yi es la fracción molar de las especies i $\left(\mathrm{CO}, \mathrm{H}_{2}\right.$ y $\left.\mathrm{CH}_{4}\right)$ del gas seco (DG).

\section{Resultados}

El modelo de gasificación ha sido validado con los resultados experimentales de Jayah et. al. El porcentaje de error promedio de la validación se calculó de la siguiente manera:

$\%$ Error $=\left|\frac{\text { Valor experimental }- \text { Valor predicho por modelo }}{\text { Valor experimental }}\right| * 100 \%$

Una comparación entre los valores obtenidos por Jayah y los resultados obtenidos por el modelo Roy [26] se muestra en la tabla 9, donde el valor de error promedio más alto es $17.81 \%$ perteneciente al fragmento de $4.4 \mathrm{~cm}$ y esto sucede cuando todos los factores que multiplican a $\mathrm{K}_{2}, \mathrm{~K}_{\mathrm{R} 3}, \mathrm{r}_{\mathrm{R} 1}$ y $\mathrm{r}_{\mathrm{R}}$ son igual a 1.

Cuando se realiza los nuevos cambios para minimizar los porcentajes de error promedio de validación se realiza la siguiente Tabla 10 , donde se muestra el factor que multiplica la constante de equilibrio químico $\left(\mathrm{K}_{2}\right.$ y $\left.\mathrm{K}_{\mathrm{R} 3}\right)$ de la reacción de metanización que ocurrió tanto en la zona de oxidación como en la reducción, cuyo valor $(=1.5)$ permanece constante para todos los fragmentos de biomasa. El factor que multiplica la velocidad cinética de la reacción de Boudouard (R1) presenta cambios crecientes ( 3 a 4 veces mayores que el fragmento menor) y el factor para la reacción de cambio de agua-gas (R2) presenta cambios decrecientes (centésima a milésima parte del fragmento menor) cuando se aumenta el diámetro del fragmento.

Los valores de los errores de promedios con respecto a los tamaños de los fragmentos de biomasa disminuyen cuando se considera la modificación del modelo de Roy [26] como se puede ver en la Tabla 11. Una explicación es la conversión de carbón donde los fragmentos más pequeños tienen una mayor probabilidad de convertirse en un gas completo antes de que comience la conversión lenta, debido a su tamaño y a las limitaciones de difusión durante el proceso de reacción. Como resultado de una conversión de char más rápida, los fragmentos más pequeños aumentan la conversión de manera más eficiente en comparación con los fragmentos más grandes [40].

Tabla 10: Parámetros modificados de las constantes de equilibrio y de las velocidades cinéticas.

\begin{tabular}{|c|c|c|c|c|c|}
\hline $\begin{array}{c}\text { Diámetro } \\
\text { del } \\
\text { fragmento } \\
(\mathbf{c m})\end{array}$ & $\begin{array}{c}\text { Prueba } \\
\mathbf{N}^{\mathbf{0}}\end{array}$ & $\begin{array}{c}\text { Factor a } \\
\mathbf{K}_{\mathbf{2}}\end{array}$ & $\begin{array}{c}\text { Factor a } \\
\mathbf{K}_{\mathbf{R} 3}\end{array}$ & $\begin{array}{c}\text { Factor a } \\
\mathbf{~} \mathbf{R}_{\mathbf{R}}\end{array}$ & $\begin{array}{c}\text { Factor a } \\
\mathbf{r}_{\mathbf{R} 2}\end{array}$ \\
\hline \multirow{3}{*}{3.3} & 1 & 1.5 & 1.5 & 1 & 1 \\
\cline { 2 - 6 } & 2 & 1.5 & 1.5 & 1 & 1 \\
\cline { 2 - 6 } & 3 & 1.5 & 1.5 & 1 & 1 \\
\hline \multirow{3}{*}{4.4} & 4 & 1.5 & 1.5 & 4 & 0.01 \\
\cline { 2 - 6 } & 5 & 1.5 & 1.5 & 4 & 0.01 \\
\cline { 2 - 6 } & 6 & 1.5 & 1.5 & 4 & 0.01 \\
\hline \multirow{3}{*}{5.5} & 7 & 1.5 & 1.5 & 3 & 0.001 \\
\cline { 2 - 6 } & 8 & 1.5 & 1.5 & 3 & 0.001 \\
\cline { 2 - 6 } & 9 & 1.5 & 1.5 & 3 & 0.001 \\
\hline
\end{tabular}

El modelo modificado se utiliza para simular el efecto de algunos parámetros operativos en la conversión y la composición del gas a la salida del reactor considerando la velocidad de flujo del bagazo seco igual a $20 \mathrm{~kg} / \mathrm{h}$, los porcentajes de nitrógeno y azufre se agregarán al porcentaje de oxígeno ya que estos elementos están presentes en pequeñas cantidades.

La relación de equivalencia ER es un factor vital en términos de temperatura de gasificación y conversión de carbón en el gasificador de biomasa. 
Tabla 9: Error promedio del modelo de Roy.

\begin{tabular}{|c|c|c|c|c|c|c|c|c|}
\hline $\begin{array}{c}\text { Diámetro del } \\
\text { fragmento } \\
(\mathbf{c m})\end{array}$ & $\begin{array}{c}\text { Prueba } \\
\mathbf{N}^{\mathbf{0}}\end{array}$ & Comparación & $\begin{array}{c}\mathrm{H}_{2} \\
(\%)\end{array}$ & $\begin{array}{l}\text { CO } \\
(\%)\end{array}$ & $\begin{array}{l}\mathrm{CO}_{2} \\
(\%)\end{array}$ & $\begin{array}{l}\mathrm{CH}_{4} \\
(\%)\end{array}$ & $\begin{array}{l}\mathrm{N}_{2} \\
(\%)\end{array}$ & $\begin{array}{c}\text { Error } \\
\text { promedio }(\%)\end{array}$ \\
\hline \multirow{9}{*}{3.3} & \multirow{3}{*}{1} & Jayah & 17.2 & 19.6 & 9.9 & 1.4 & 51.9 & \\
\hline & & Modelo Roy & 19.37 & 18.29 & 10.99 & 1.21 & 50.15 & \\
\hline & & $\%$ Error & 12.60 & 6.69 & 11.02 & 13.85 & 3.38 & 9.51 \\
\hline & \multirow{3}{*}{2} & Jayah & 18.3 & 20.2 & 9.7 & 1.1 & 50.7 & \\
\hline & & Modelo Roy & 18.18 & 19.22 & 10.32 & 1.12 & 51.16 & \\
\hline & & $\%$ Error & 0.65 & 4.87 & 6.42 & 1.80 & 0.91 & 2.93 \\
\hline & \multirow{3}{*}{3} & Jayah & 17.2 & 19.4 & 9.7 & 1.1 & 52.6 & \\
\hline & & Modelo Roy & 17.44 & 19.66 & 10.03 & 1.04 & 51.82 & \\
\hline & & $\%$ Error & 1.42 & 1.35 & 3.39 & 5.17 & 1.48 & 2.56 \\
\hline \multirow{9}{*}{4.4} & \multirow{3}{*}{4} & Jayah & 17 & 18.4 & 10.6 & 1.3 & 52.7 & \\
\hline & & Modelo Roy & 19.83 & 19.07 & 10.56 & 1.20 & 49.33 & \\
\hline & & $\%$ Error & 16.68 & 3.66 & 0.40 & 7.42 & 6.39 & 6.91 \\
\hline & \multirow{3}{*}{5} & Jayah & 13.2 & 19.7 & 10.8 & 1.3 & 55 & \\
\hline & & Modelo Roy & 18.46 & 19.20 & 10.32 & 1.15 & 50.86 & \\
\hline & & $\%$ Error & 39.85 & 2.53 & 4.43 & 11.27 & 7.52 & 13.12 \\
\hline & \multirow{3}{*}{6} & Jayah & 12.5 & 18.9 & 8.5 & 1.2 & 59.1 & \\
\hline & & Modelo Roy & 17.81 & 19.87 & 9.92 & 1.06 & 51.34 & \\
\hline & & $\%$ Error & 42.47 & 5.14 & 16.71 & 11.59 & 13.13 & 17.81 \\
\hline \multirow{8}{*}{5.5} & \multirow{3}{*}{7} & Jayah & 15.5 & 19.1 & 11.4 & 1.1 & 52.9 & \\
\hline & & Modelo Roy & 20.57 & 19.34 & 10.47 & 1.23 & 48.39 & \\
\hline & & $\%$ Error & 32.73 & 1.23 & 8.20 & 12.00 & 8.52 & 12.54 \\
\hline & \multirow{3}{*}{8} & Jayah & 12.7 & 22.1 & 10.5 & 1.3 & 53.4 & \\
\hline & & Modelo Roy & 18.83 & 19.87 & 9.92 & 1.16 & 50.21 & \\
\hline & & $\%$ Error & 48.24 & 10.07 & 5.48 & 10.69 & 5.97 & 16.09 \\
\hline & \multirow[b]{2}{*}{9} & Jayah & 13 & 19.1 & 10.7 & 1.2 & 56 & \\
\hline & & Modelo Roy & 17.38 & 20.06 & 9.76 & 1.03 & 51.76 & \\
\hline
\end{tabular}

Su valor se define en un rango de 1 a 6 , por lo tanto, cada uno de estos términos se evaluará dentro del rango de contenido de humedad de 0 a $40 \%$.

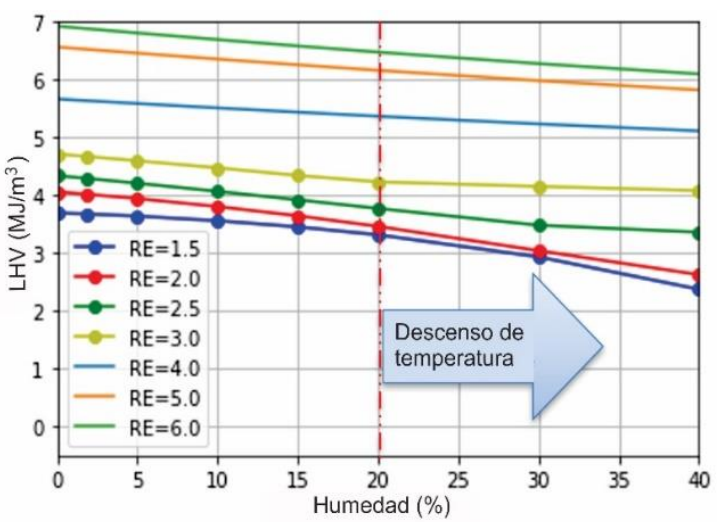

Figura 2: Valores térmicos más bajos con respecto al contenido de humedad de las proporciones de equivalencias del bagazo de caña de azúcar
Para este caso, los factores que multiplican $\mathrm{K}_{2}, \mathrm{~K}_{\mathrm{R} 3}$, $r_{R 1}$ y $r_{R 2}$ serán los correspondientes al fragmento 3,3 $\mathrm{cm}$. El programa registra una serie de temperaturas dentro del rango de $1000^{\circ} \mathrm{K}$ a $2500^{\circ} \mathrm{K}$. Sin embargo, se seleccionarán temperaturas inferiores a $1700^{\circ} \mathrm{K}$ debido a la resistencia máxima de los termopares tipo $\mathrm{K}$ que se encuentran en la zona de oxidación del gasificador. La figura 2 muestra que el LHV aumenta a medida que aumenta ER.

Las curvas de LHV muestran una disminución cuando aumenta la humedad. Además, hay curvas de LHV que están muy cerca en el rango de MC de 0 a $20 \%$, lo que indica que la composición de los gases es muy similar a la salida del gasificador.

La línea roja punteada indica la cantidad máxima de humedad de bagazo que podría ingresar al gasificador. 
Tabla 11: Error promedio del modelo modificado.

\begin{tabular}{|c|c|c|c|c|c|c|c|c|}
\hline $\begin{array}{c}\text { Diámetro del } \\
\text { fragmento }\end{array}$ & $\begin{array}{c}\text { Prueba } \\
\text { No }^{o}\end{array}$ & Comparación & $\begin{array}{c}\mathrm{H}_{2} \\
(\%)\end{array}$ & $\begin{array}{l}\mathrm{CO} \\
(\%)\end{array}$ & $\begin{array}{l}\mathrm{CO}_{2} \\
(\%)\end{array}$ & $\begin{array}{l}\mathrm{CH}_{4} \\
(\%)\end{array}$ & $\begin{array}{l}\mathrm{N}_{2} \\
(\%)\end{array}$ & $\begin{array}{c}\text { Error promedio } \\
(\%)\end{array}$ \\
\hline \multirow{9}{*}{3.3} & \multirow[t]{3}{*}{1} & Jayah & 17.2 & 19.6 & 9.9 & 1.4 & 51.9 & \\
\hline & & Modelo modificado & 19.30 & 18.30 & 10.99 & 1.24 & 50.16 & \\
\hline & & $\%$ Error & 12.20 & 6.61 & 11.05 & 11.12 & 3.36 & 8.87 \\
\hline & \multirow[t]{3}{*}{2} & Jayah & 18.3 & 20.2 & 9.7 & 1.1 & 50.7 & \\
\hline & & Modelo modificado & 18.10 & 19.24 & 10.32 & 1.17 & 51.17 & \\
\hline & & $\%$ Error & 1.09 & 4.77 & 6.44 & 6.30 & 0.93 & 3.91 \\
\hline & \multirow[t]{3}{*}{3} & Jayah & 17.2 & 19.4 & 9.7 & 1.1 & 52.6 & \\
\hline & & Modelo modificado & 17.35 & 19.69 & 10.03 & 1.10 & 51.83 & \\
\hline & & $\%$ Error & 0.87 & 1.48 & 3.41 & 0.20 & 1.46 & 1.48 \\
\hline \multirow{9}{*}{4.4} & \multirow[t]{3}{*}{4} & Jayah & 17 & 18.4 & 10.6 & 1.3 & 52.7 & \\
\hline & & Modelo modificado & 16.35 & 18.78 & 9.86 & 1.31 & 53.70 & \\
\hline & & $\%$ Error & 3.82 & 2.08 & 6.95 & 0.40 & 1.89 & 3.03 \\
\hline & \multirow[t]{3}{*}{5} & Jayah & 13.2 & 19.7 & 10.8 & 1.3 & 55 & \\
\hline & & Modelo modificado & 14.09 & 18.53 & 9.63 & 1.26 & 56.48 & \\
\hline & & $\%$ Error & 6.74 & 5.93 & 10.79 & 3.00 & 2.70 & 5.83 \\
\hline & \multirow[t]{3}{*}{6} & Jayah & 12.5 & 18.9 & 8.5 & 1.2 & 59.1 & \\
\hline & & Modelo modificado & 11.98 & 19.23 & 8.85 & 1.15 & 58.78 & \\
\hline & & $\%$ Error & 4.16 & 1.77 & 4.15 & 3.86 & 0.54 & 2.90 \\
\hline \multirow{9}{*}{5.5} & \multirow[t]{3}{*}{7} & Jayah & 15.5 & 19.1 & 11.4 & 1.1 & 52.9 & \\
\hline & & Modelo modificado & 17.63 & 18.46 & 10.24 & 1.33 & 52.34 & \\
\hline & & $\%$ Error & 13.77 & 3.34 & 10.19 & 20.69 & 1.06 & 9.81 \\
\hline & \multirow[t]{3}{*}{8} & Jayah & 12.7 & 22.1 & 10.5 & 1.3 & 53.4 & \\
\hline & & Modelo modificado & 14.75 & 18.45 & 9.74 & 1.26 & 55.80 & \\
\hline & & $\%$ Error & 16.12 & 16.53 & 7.26 & 2.77 & 4.50 & 9.43 \\
\hline & \multirow[t]{3}{*}{9} & Jayah & 13 & 19.1 & 10.7 & 1.2 & 56 & \\
\hline & & Modelo modificado & 11.02 & 18.13 & 9.31 & 1.10 & 60.44 & \\
\hline & & $\%$ Error & 15.22 & 5.10 & 12.95 & 7.95 & 7.92 & 9.83 \\
\hline
\end{tabular}

La Tabla 12 muestra las posibles temperaturas de trabajo dentro del gasificador, a la salida de la zona de pirooxidación. Sin embargo, hemos seleccionado

solo aquellos que tienen el LHV más alto y la menor cantidad de carbón en el colector.

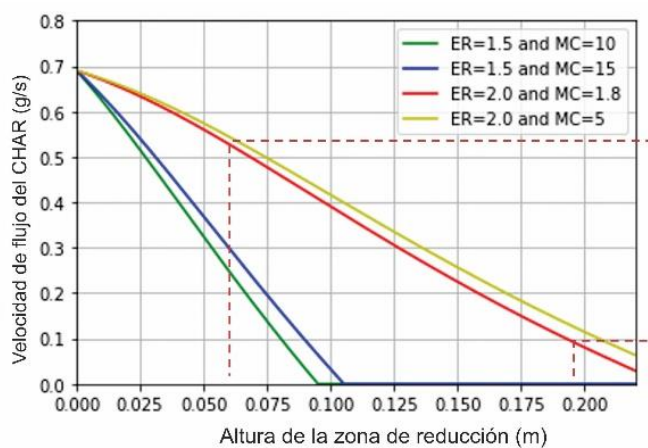

(a)

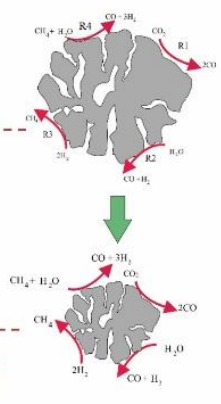

(b)
Figura 3: (a) Velocidad de flujo de carbón a lo largo de la altura de la zona de reducción y (b) imagen de partículas de carbón con las 4 reacciones ocurridas en la zona de reducción.

Cuando el contenido de humedad del bagazo es alto, se gasta mucho calor generado en el gasificador para vaporizar la humedad $y$ sobrecalentar el vapor. como resultado, la temperatura de funcionamiento del gasificador disminuye. Asimismo, la tabla 13 muestra la cantidad de gas pobre que se obtiene por kilogramo de biomasa seca que ingresa al gasificador y el cálculo estimado del potencial energético del bagazo de caña de azúcar en Perú, considerando que la densidad promedio del gas pobre es $1.3 \mathrm{Kg}$ $/ \mathrm{m}^{3}[41]$.

La figura 3. muestra el comportamiento de la conversión del CHAR a lo largo de la zona de reducción, en donde podemos deducir que para $E R<2$, la cantidad de CHAR se consume por completo, por lo que no habría más reacciones cinéticas y solo obtendríamos cenizas en el colector del gasificador.

Para que esto suceda, la zona de pirooxidación debe tener una temperatura superior a $1700^{\circ} \mathrm{K}$, pero esto no es posible debido a las condiciones de infraestructura del gasificador.

En este sentido, la temperatura alcanzada a la salida de la zona de pirooxidación debe encontrarse dentro de un rango que varíe entre $1300^{\circ} \mathrm{K}$ a $1550^{\circ} \mathrm{K}$ [30]. De esta manera nos aseguramos de que la cantidad de CHAR en el colector del gasificador sea la mínima posible para diferentes valores de ER y contenido de humedad de bagazo. 
Tabla 12: Resultados de la simulación.

\begin{tabular}{|c|c|c|c|c|c|c|c|c|c|c|c|c|}
\hline $\begin{array}{l}\text { Humedad } \\
(\%)\end{array}$ & \multicolumn{4}{|c|}{$\begin{array}{l}\text { Temperatura de pirooxidación } \\
(\mathrm{K})\end{array}$} & \multicolumn{4}{|c|}{$\begin{array}{c}\text { LHV } \\
\left(\mathrm{MJ} / \mathrm{m}^{3}\right)\end{array}$} & \multicolumn{4}{|c|}{$\begin{array}{l}\text { Masa final de char } \\
(\mathrm{mg} / \mathrm{s})\end{array}$} \\
\hline 1.8 & 1796.3 & 1530.7 & 1318.5 & 1144.7 & 3.67 & 4.01 & 4.28 & 4.65 & 0 & 31.8 & 447.6 & 631.2 \\
\hline 10 & 1722.5 & 1456.1 & 1245.8 & 1076.8 & 3.56 & 3.8 & 4.06 & 4.46 & 0 & 128.4 & 517.2 & 650.532 \\
\hline 15 & 1673.6 & 1407 & 1198.3 & 1033.3 & 3.45 & 3.64 & 3.91 & 4.32 & 0 & 199.2 & 560.16 & 653.76 \\
\hline 20 & 1621.3 & 1354.8 & 1148.2 & 1000 & 3.31 & 3.45 & 3.76 & 4.21 & 0 & 276 & 598.8 & 648.564 \\
\hline
\end{tabular}

Tabla 13: Potencial energético del Perú estimado.

\begin{tabular}{|c|c|c|c|c|c|c|c|c|}
\hline $\begin{array}{c}\text { Humedad } \\
(\%)\end{array}$ & \multicolumn{4}{|c|}{ Kg de PG/Kg de DB } & \multicolumn{4}{c|}{$\begin{array}{c}\text { Potencial Energético } \\
\text { (TJ/año) }\end{array}$} \\
\cline { 2 - 9 } & ER=1.5 & ER=2.0 & ER=2.5 & ER=3.0 & ER=1.5 & ER=2.0 & ER=2.5 & ER=3.0 \\
\hline $\mathbf{1 . 8}$ & 4.61 & 3.46 & 2.82 & 2.45 & 23614.1 & 19396.6 & 16843.7 & 15882.4 \\
\hline $\mathbf{5}$ & 4.60 & 3.46 & 2.82 & 2.46 & 23375.3 & 19008.6 & 16518.3 & 15700.9 \\
\hline $\mathbf{1 0}$ & 4.58 & 3.44 & 2.81 & 2.47 & 22781.1 & 18247.2 & 15957.5 & 15390.3 \\
\hline $\mathbf{1 5}$ & 4.56 & 3.42 & 2.81 & 2.49 & 21947.2 & 17387.5 & 15368.0 & 15026.5 \\
\hline $\mathbf{2 0}$ & 4.52 & 3.40 & 2.82 & 2.51 & 20907.0 & 16367.3 & 14797.3 & 14770.8 \\
\hline
\end{tabular}

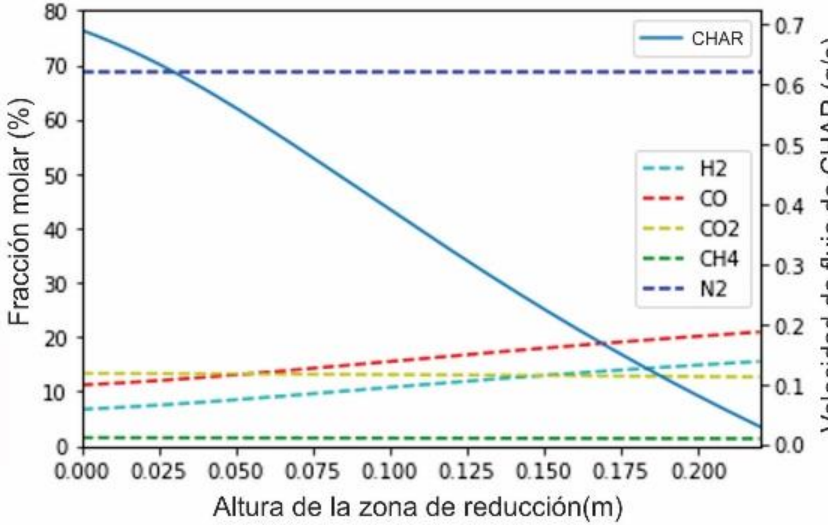

(a)

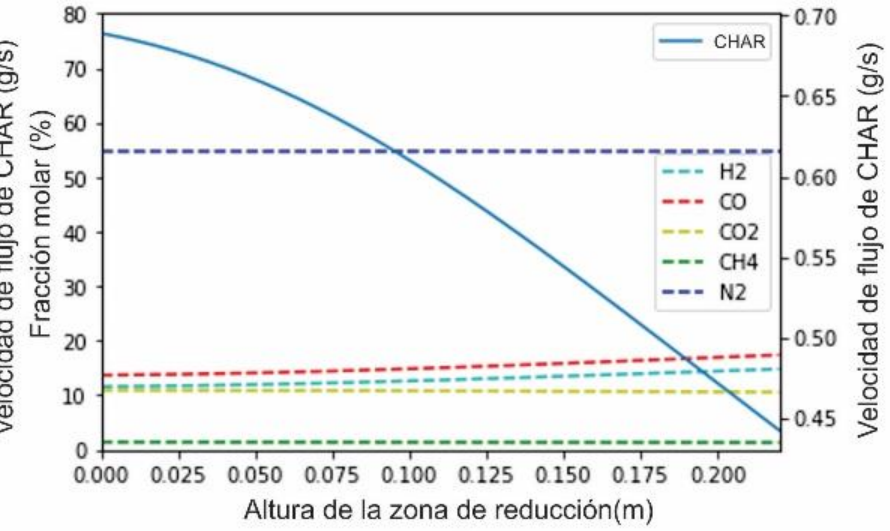

(b)

Figura 4: Composición del gas y flujo del CHAR.

Por lo tanto, considerando la temperatura más alta a la salida de la zona de pirooxidación del gasificador, obtenemos un potencial de energía estimado de 19396.6 TJ / año cuando ER es 2 y MC es 1.8 , lo que puede ser conveniente debido a la baja cantidad de CHAR que se encontraría en el colector $(31.8 \mathrm{mg} / \mathrm{s})$.

A diferencia de esto, a temperaturas más bajas, el potencial de energía es 16843.7 TJ / año cuando ER es 2.5 y $\mathrm{MC}$ es 1.8, y la cantidad de CHAR encontrada es $447 \mathrm{mg} / \mathrm{s}$. En la Figura 4(a), para ER $=2$, la concentración de $\mathrm{H}_{2}$ aumenta aproximadamente $131 \%$ cuando la altura de la zona de reducción aumenta de 0 a $0.22 \mathrm{~m}$. En la figura 4(b), el aumento correspondiente de la concentración de $\mathrm{ER}=2.5$ es aproximadamente $28 \%$. Por otro lado, la fracción molar de CO aumenta en un $87.1 \%$ a $E R=2.0$ y en un $27.4 \%$ a $\mathrm{ER}=2.5$. El contenido de $\mathrm{CH}_{4}$ muestra una naturaleza diferente de la variación en las dos relaciones de equivalencia diferentes: la fracción molar de $\mathrm{CH}_{4}$ disminuye en un $12 \%$ a $\mathrm{ER}=2.0$ y en un $5.5 \%$ a $E R=2.5$.

\section{Conclusiones}

En este trabajo se estimó el potencial energético del gas pobre obtenido de la gasificación del bagazo de caña de azúcar de manera computacional, producido por las agroindustrias azucareras del Perú, obteniendo un valor máximo de 19396.6 TJ / año y un valor mínimo de 16843.7 TJ / año. Por lo tanto, el potencial energético promedio obtenido con gasificadores de tiro descendente de garganta para el año 2017 podría estimarse en 18120.15TJ/año a 
partir de 11127473 Toneladas / año de una producción total de caña de azúcar.

Para alcanzar estos valores, se ha utilizado un modelo de gasificación propuesto por Roy. Después de haber modificado las constantes de equilibrio en la zona de oxidación y las velocidades cinéticas en la zona de reducción; los componentes del gas pobre a diferentes tamaños de fragmento fueron validadas con los datos experimentales de Jayah obteniendo mejores predicciones. La distribución de temperaturas y concentraciones de especies en el gasificador se ha predicho en el modelo. Se ha considerado un factor de reactividad de carbón constante $(\mathrm{CRF}=100)$ después de validar las predicciones con los resultados experimentales de la literatura. El modelo fue desarrollado en un lenguaje de programación llamado Python versión 3.7 en un entorno de trabajo interactivo Jupyter Notebook.

La zona de pirooxidación del gasificador se ha resuelto teniendo en cuenta el equilibrio químico de la especie. Sin embargo, se han adoptado reacciones químicas a velocidades finita basadas en la cinética de las reacciones de reducción para la solución en la zona de reducción. El valor calorífico inferior del gas pobre aumenta con el aumento en la relación de equivalencia. El aumento en el contenido de humedad del bagazo deteriora la calidad del gas pobre y su valor de calentamiento disminuye. Para el potencial energético estimado en el Perú se consideró que toda la cantidad de bagazo disponible en el Perú se utilizaría en gasificadores de tiro descendente para obtener gas pobre $y$ generar calor y electricidad en comunidades rurales ubicadas en las cercanías de plantas de agroindustrias azucareras.

En un trabajo futuro, se considerará una variedad de biomasas con un alto contenido de cenizas para estudiar el efecto de las cenizas en la gasificación, como se ha visto en este documento con la biomasa de bagazo y su relación con la referencia reportada en la literatura.

\section{Agradecimientos}

Los autores desean expresar su sincero agradecimiento al laboratorio de la Facultad de Petroquímica por haber realizado el análisis de las muestras. CZ desea agradecer a FONDECYT (Convenio $\mathrm{n}^{\circ}$ 207-2015-FONDECYT) por su beca de doctorado.

\section{Referencias}

[1] N. Aragon et al., Sustainable land management for bioenergy crops, Energy Procedia (2017) 125-379.

[2] D. Silva et al., Life cycle assessment of the sugarcane bagasse electricity generation in Brazil, Renewable Sustainable Energy (2014) 532-547.

[3] M. Amutio et al., Fast Characterization of biomass fuels by thermogravimetric analysis, Fuel (2014) 744-751.

[4] Ministerio de Agricultura y Riego, Serie de Estadísticas de Producción Agrícola (MINAGRI, Perú, 2019)

[5] L. Paula, Characterization of Residues from Plant Biomass for Use in Energy Generation, CERNE (2011) 237-246.

[6] L. Valadez et al., Valorization of cacao pod husk through supercritical fluid extraction of phenolic compounds, Supercrit Fluids (2018) 99-105.

[7] Ministerio de Energía y Minas, Plan Energético Nacional 2014 - 2025, (MINEM, Perú, 2019).

[8] R. García et al., Characterization of Spanish biomass wastes for energy use, Bioresource Technology (2012) 249-258.

[9] A. Viviana et al., Caracterización termogravimétrica de carbonizados de residuos provenientes de la uva Isabella (Vitis labrusca), Científica Ingeniería y Desarrollo 36 (2018) 2.

[10] R. García et al., Biomass proximate analysis using thermogravimetry. Bioresource. Technology. (2013) 1-4.

[11] Ministerio de Energía y Minas, Balance Nacional de Energía 2016 (MINEM, Perú, 2019).

[12] $\mathrm{R}$ Chowdhury, $M$ Chakravarty and $\mathrm{P}$. Bhattacharya. Modelling and simulation of an updraft moving bed gasifier using rice husk as fuel material. International Journal of Energy Research (2007) 593-602.

[13] P. Krishnamoorthy, S. Seetharamu and M. Bhatt. Development of a novel updraft multifuel biomass gasifier. International Journal of Energy Research (2007) 377390.

[14] P. McKendry, Energy production from biomass: gasification technologies, Bioresource Technology 83 (2002) 55-63.

[15] S. Dasappa et al. Biomass gasification technology, a route to meet energy needs. Indian Academy of Science 87 (2004) 908916. 
[16] C. Altafini, P. Winnder and R. Barreto, Prediction of the working parameters of a wood waste gasifier through an equilibrium model, Energy Conversion and Management (2003) 2763-2777.

[17] A. Melgar et al., Thermochemical equilibrium modelling of a gasifying process, Energy Conversion and Management (2007) 59-67.

[18] A. Sharma et al., Equilibrium modeling of global reduction reactions for a downdraft gasifier, Energy Conversion and Management (2008) 832-842.

[19] S. Jarungthammachote and A. Dutta Thermodynamic equilibrium model and second law analysis of a downdraft waste gasifier, Energy (2007) 1660-1669.

[20] M. Ruggiero and G. Manfrida, An equilibrium model for biomass gasification processes, Renewable Energy (1999) 1106-1109.

[21] Z. Zainal et al., Prediction of performance of a downdraft gasifier using equilibrium modeling for different biomass materials, Energy Conversion and Management (2001)1499-1515.

[22] D. Giltrap, R. McKibbin and G. Barnes, A steady state model of gas-char reactions in a downdraft gasifier, Solar Energy (2003) 85-91.

[23] B. Babu and P. Sheth, Modeling and simulation of reduction zone of downdraft biomass gasifier: effect of char reactivity factor, Energy Conversion and Management (2006) 2602-2611.

[24] A. Sharma, Equilibrium and kinetic modeling of char reduction reactions in a downdraft biomass gasifier: a comparison, Solar Energy (2008) 918-928.

[25] A. Kuchling, Interview with Guido van Rossum, Linux Journal,(1998).

[26] Jupyter Project and Community, (2019).

[27] Y. Wang and C. Kinoshita, Kinetic model of biomass gasification, Solar Energy (1993)19-25.

[28] C. Serrato, Metodología para el cálculo de la energía extraída de la biomasa en el Departamento de Cundinamarca, Tesis pregrado, Universidad Francisco José de Caldas, 2016.

[29] P. Roy, A. Datta and N. Chakraborty, Modeling of a downdraft biomass gasifier with finite rate kinetics in the reduction zone, International Journal of Energy Research 33 (2009) 833-851.
[30] T. Jayah et al., Computer simulation of a downdraft wood gasifier for tea drying. Biomass and Bioenergy (2003) 459-469.

[31] A. Varma and P. Mondal, Physicochemical Characterization and Pyrolysis Kinetic Study of Sugarcane Bagasse Using Thermogravimetric Analysis, Journal Energy Resource Technol 138 (2016) 5.

[32] E. Virmond et al., Valorization of agroindustrial solid residues and residues from biofuel production chains by thermochemical conversion: A review, citing brazil as a case study, Brazilian Journal Chemistry Engennering 30 (2013) 197-229.

[33] R. Probstein and R. Hicks, Synthetic fuel edited by Mc Graw Hill (New York ,1982).

[34] J. Bonnie and McBride, Coefficients for calculating thermodynamic and transport properties of Individual species, Report NASA Technical № 4513, 1993.

[35] W. Weng, Y. Hasemi and W. Fana, Predicting the pyrolysis of wood considering char oxidation under different ambient oxygen concentrations, Combustion and Flame (2006) 723-729.

[36] P. Liang, Z. Wang Z and J. Bi , Simulation of coal pyrolysis by solid heat carrier in a moving-bed pyrolyzer, Fuel (2008) 435-442.

[37] A. Sharma. Modeling and simulation of a downdraft biomass gasifier, Energy Conversion Management (2011) 13861396.

[38] S. Hameed et al., Kinetic modeling of reduction zone in biomass gasification, Energy Conversion Management (2014) 367-373.

[39] Lars Waldheim, Heating value of gases from biomass gasification, Report № 202001 .

[40] J. Chen, Kinetic engineering modelling of cocurrent moving bed gasification reactors for carbonaceous material, D. Ph. Thesis, Cornell University, 1987.

[41] G. Maschio, Production of Syngas from Biomass, Bioresource technology 83 (1993) 119-126.

E-mails: czavalai@uni.edu.pe vpretell@fip.uni.edu.pe 